\title{
Impact of default rate of antiretroviral use on viral load among HIVIAIDS patients in Tamale and Sunyani
}

\begin{abstract}
Background: Highly Active Antiretroviral Therapy (HAART) for persons living with HIV/ AIDS (PLWHA) is the gold standard for the management of HIV patients. Purportedly, patients who are not on HAART or defaulted from its use have adverse effects compared to those who adhere to the intake of HAART. Compliant individuals have improved quality of life and show healthy hematological parameters and HIV load as compared to HAART naïve individuals and defaulters. Supplementary and supportive treatment is crucial in HIV/ AIDS patients on patients on antiretroviral therapy?
\end{abstract}

Objective: This study was conducted to assess the consistency and default of HAART intake and other supportive treatments and its relationship with viral load on hematological parameters in two different geographical sites.

Methods: Ethical clearance was obtained from Navrongo Health Research Centre Questionnaires were administered to participants for their consent, demographic data, consistency of taking antiretrovirals, and types of antiretrovirals used. Full blood count and HIV load tests were carried out using Urit 5250 and Cobas Taqman / Cobas ampliprep fully automated analysers respectively.

Results: Defaulters had a significant ( $\mathrm{p}$ value $=0.003$ ) rise in their viral load (845334.38 \pm 409285.62$)$ copies $/ \mathrm{mL}$ in comparison to adherents in HAART intake $49571.69 \pm 30548.89)$ copies $/ \mathrm{mL}$. The hemoglobin level $(10.51 \pm 0.60 \mathrm{~g} / \mathrm{dL})$ of defaulters was significantly $(\mathrm{p}$-value $=0.007)$ lower than that of adherent $(12.04 \pm 0.17 \mathrm{~g} / \mathrm{dL})$. The default rates in the two study sites were close $(9.8 \%$ in Sunyani and $9.4 \%$ in Tamale).

Conclusion: Antiretroviral default does not only lead to an increase in HIV load but also causes a reduction in hemoglobin levels. Hematinics supplementation therapy may help normalize hematological parameters in HIV infection.
Volume 8 Issue 3 - 202 I

\author{
Abban EF,' Abugri J, ${ }^{2}$ Mohammed F,' Asigri F,' \\ Addo A, 'A bass AK, ${ }^{3}$ Wezena CA, ${ }^{4}$ Helegbe \\ $\mathrm{GK}^{5}$ \\ 'University for Development Studies Tamale, Ghana \\ ${ }^{2}$ Department of Applied Chemistry and Biochemistry, C. \\ K. Tedam University of Technology and Applied Sciences \\ ${ }^{3}$ Navrongo, Ghana, Abass Abdul-Kasim, Pubblic Health Reference \\ Laboratory,Tamale, Ghana abass.komei@yahoo.com \\ ${ }^{4}$ Department of Microbiology, School of Biosciences, Nyankpala \\ Campus, University for Development Studies, Ghana \\ ${ }^{5}$ Department of Biochemistry and Molecular Medicine, School \\ of Medicine, University for Development Studies Tamale, Ghana
}

Correspondence: James Abugri, Department of Applied Chemistry and Biochemistry, C. K. Tedam University of Technology and Applied Sciences, Navrongo, Ghana, Email jabugri@cktutas.edu.gh

Received: April 20, 2021 | Published: June 15, 2021

\section{Introduction}

The human immune deficiency virus (HIV) adversely affects the body's immune defense especially CD 4 cells and spreads by exchange of a variety of body fluids such as blood, breastmilk, semen, rectal fluid, and vaginal secretions from an infected person. ${ }^{1}$ This damage to the immune system is challenging and the body becomes less immuno-competent and thus susceptible to opportunistic infections and hematological complications. ${ }^{2}$ Research shows that timely access to antiretroviral therapy (ART) by people living with HIV reduces their transmission of the virus and reduces morbidity and mortality ${ }^{3}$ associated with the disease (HIV/AIDS) it causes. ${ }^{4-6}$ In effect, ART adherence improves productivity and quality of life..$^{4,7,8}$ Default in antiretroviral therapy (ART) results when an HIV patient without a relatively stable viral load misses intake of ARTs for a month or when an infected person with a relatively stable viral load misses ART for three months. ${ }^{9}$ In Ghana, experts in the study sites indicated that when a patient who has exhausted the medication does not take reimbursement of the ART for two or more clinic days; it is classified as a default.

While studies in Ghana have spelt out some of the hindrances to accessing ART, enough attention has not been given to the perspectives of PLWHA in this respect. Understanding the conditions of PLWHA and the barriers they face in getting access to ART is important to implement initiatives to redress these barriers and to better improve access and adherence to HAART ${ }^{10}$ interrogated HIV-positive persons' motivation for taking ART. Respondents intimated that ART will suppress their HIV load and make them healthy and ensure a prolonged life. Female respondents further indicated consistently that ART will prevent infection in their unborn children and avoid death. ${ }^{4}$ However, $63.3 \%$ of respondents identified seven major factors as barriers that hinder their access to ART and directly contribute to their default of ART. These barriers included high costs associated with access to ARTs, delays at treatment centers, inadequate supply of drugs, stigma, ${ }^{11}$ side effects of the ART, job insecurity arising out of absenteeism, and inaccessibility of treatment centers. ${ }^{4}$ Delays during hospital/clinic reviews also demotivated people living with HIV from frequent visits to access ART in Ghana. ${ }^{12}$

An earlier study in Ghana on adherence to ART among PLWHA and their hematological parameters revealed that a six-month adherence to ART led to a reduction in anemia, leucopenia, and thrombocytopenia. This outcome reflects the long-term potency of ART. However, this depended on consistency in adherence to ART treatment ${ }^{13}$ in the long term. ${ }^{9}$ Thrombocytopenia, anemi, ${ }^{14}$ leucopenia, and lymphocytosis have been associated with a high prevalence of deviation in hematological parameters, with anemia. ${ }^{15}$ This also investigated the relationship between hematologic parameters and ART default rate.

Previous studies show that administering ART, particularly zidovudine (AZT) therapy, leads to anemia with a significant reduction in hemoglobin level among HIV patients. ${ }^{16}$ Viral load 
is the gold standard method to diagnose ART failure. ${ }^{17}$ However, it is generally not accessible in resource-limited settings ${ }^{18}$ as in most clinics in Ghana. Co-infection with tuberculosis is associated with reduced immunity against HIV,${ }^{17}$ and combined ART/Anti-TB therapy is feasible and more effective. ${ }^{19,20} \mathrm{In}$ vitro studies indicate that tuberculosis spurs HIV replication by activating CD4 T lymphocytes and macrophages harboring latent HIV. The onset of tuberculosis in HIV-infected patients causes a marked release of proinflammatory cytokines that activate lymphocytes and macrophages. This results in increases in the viral load ${ }^{17,21}$ and death rate. ${ }^{22}$

\section{Methods}

\section{Study area and design}

This prospective study was conducted from October 2018 to July 2019 in the Sexually Transmitted Infection Clinics of the Tamale Teaching Hospital (TTH) and the Sunyani Regional Hospital (SRH) in Northern and Bono Regions of Ghana respectively.

\section{Study population}

A total of 260 PLWHA visiting the Anti-Retroviral Therapy Units of the two hospitals were recruited for this study after obtaining ethical approval from the Institutional Review Board of the Navrongo Health Research Center. Informed consent was obtained from the participants before data and sample collection. One hundred and twenty (120) participants were recruited from Tamale Teaching Hospital while one hundred and forty (140) participants were recruited from Sunyani Regional Hospital. Participants were stratified according to the type of ART, the period of taking ART, and whether they had defaulted or not. HAART used was defined as the receipt of two nucleoside reverse transcriptase inhibitors (NRTI) and one nonnucleoside reverse transcriptase inhibitor (NNRTI) or one protease inhibitor (PI). The degree of adherence to antiretroviral therapy was categorized into always consistent, consistent sometimes, and not consistent. Always consistent was used to describe participants who were religiously following their HAART and never missed taking their drugs. Consistent sometimes was used to describe participants who missed their drug only a few times in the month but not up to a month. Not consistent or default in antiretroviral therapy was used to describe participants who missed their HAART for up to one month or more.

\section{Sample collection and preparation}

A venous blood sample $(5 \mathrm{ml})$ was drawn into an EDTA tube using the standard phlebotomy protocol for each study participant following World Health Organization (WHO) guideline. ${ }^{23}$ Full blood count (FBC) was performed after which the sample was used for the estimation of viral load. FBC was done using Urit 5250 hematology analyser. Parameters considered include white blood cells, neutrophils, lymphocytes, monocytes, eosinophils, and basophils.

The HIV load assay was conducted using COBAS AmpliPrep and COBAS TaqMan analysers according to the Centre for Disease Control and Prevention and World Health Organization. ${ }^{24}$ HIV RNA was quantitatively amplified from human plasma. An automated COBAS AmpliPrep Instrument was used to prepare and amplify sample HIV RNA while a COBAS TaqMan Analyzer was used to detect and analyze Polymerase Chain Reaction products.

\section{Statistical analysis}

Data were collected with structured questionnaires in Google form and analyzed with SPSS version 25. The variables were done using frequencies, means, and variability using standard deviation and tabulations. Tests for significance of variables using correlations and significance of the variables were carried out in multivariate analysis. A p-value of less than 0.05 was adjudged significant.

\section{Results}

\section{Types of medications used by HIV subjects}

Table 1 presents the type of antiretrovirals taken by participants to control HIV progression. These medications included anti-retroviral drugs such as Tenofovir, Lamivudine, Zidovudine, Nevirapine, Lopinavir, Abacavir, and Emtricitabine in various combinations, antibiotics such as Erythromycin and Septrin, and hematinics such as Nexcofer and Polyfer. 199 participants $(76.5 \%)$ took Tenofovir in combination with Lamivudine and Efavirenz. 27 participants (10.4\%) took Nevirapine in combination with Lamivudine and Zidovidine while 9 participants $(3.5 \%)$ took Zidovidine in combination with Lamivudine and Nevirapine. 7 participants $(2.7 \%)$ took Zidovidine in combination with Lamivudine and Lopinavir. 4 participants (1.5\%) took Tenofovir in combination with Nevirapine and Lamivudine. Other combinations of the antiretrovirals listed above were taken by only one participant. 94 participants $(47.2 \%)$ revealed they were also under antibiotic medication in addition to the ART. 89 (34.2\%) of participants also took septrin while $5(1.9 \%)$ of participants also took erythromycin. Less than half of the participants $(89,34.2 \%)$ also took one of the two kinds of hematinics. $64(25 \%)$ and $25(10 \%)$ participants, took nexcofer and polyfer respectively as hematinic supplements.

Table I Types of medications used by HIV subjects $(n=260)$

\begin{tabular}{lll}
\hline ARV drugs & Percentage (\%) & Frequency \\
\hline Lamivudine+Nevirapine+Abacavir & 0.4 & $\mathrm{I}$ \\
Lamivudine+Nevirapine+Zidovidine & 10.4 & 27 \\
Lamivudine+Nevirapine+Efavirenz & 0.4 & $\mathrm{I}$ \\
Zidovidine+Lamivudine+Lopinavir & 2.7 & 7 \\
Zidovidine+Lamivudine+Nevirapine & 3.5 & 9 \\
Tenofovir+Lamivudine+Efavirenz & 76.5 & 199 \\
Tenofovir+Lamivudine+Nevirapine & 1.5 & 4 \\
Tenofovir+Nevirapine+Abacavir & 0.4 & $\mathrm{I}$ \\
Tenofovir+Emtricitabine+Efavirenz & 0.4 & $\mathrm{I}$ \\
Tenofovir+Emtricitabine+Nevirapine & 0.4 & $\mathrm{I}$ \\
Antibiotic & Percentage & Frequency \\
Erythromycin & 1.9 & 5 \\
Septrin & 34.2 & 89 \\
Hematinic & Percentage & Frequency \\
Nexcofer & 24.6 & 64 \\
Polyfer & 9.6 & 25 \\
\hline
\end{tabular}

\section{Default rate of antiretroviral use}

Figure $1 \&$ Table 2 presents the default rate of antiretroviral uptake among PLWHA. Analyses of the adherence and default rates of antiretroviral therapy among persons living with HIV revealed that 186 participants $(71 \%)$ of participants were always consistent in their use of ARVs and took their medication religiously. 41 participants 
(16\%) were mostly consistent with their medication and missed their therapy only a few days in a month. 25 participants $(10 \%)$ were not consistent with their medication and defaulted many times in the month or were not on ART for more than three months in the year. As shown in Table 3, the majority of participants, $70 \%$ and $73 \%$, in
Sunyani and Tamale respectively religiously took their antiretroviral drugs and the HAART for PLWHA. However, 9.8\% and 9.4\% of participants in Sunyani and Tamale respectively defaulted in the HAART.

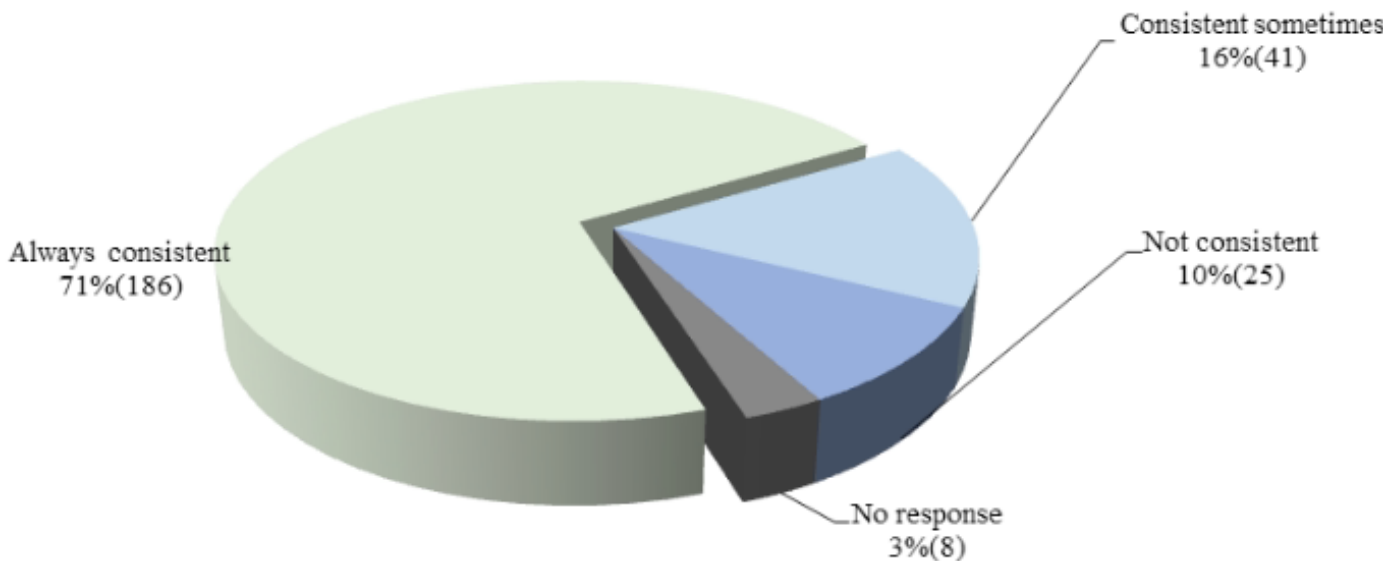

Figure I Default rate of anti-retro viral use among HIV patients.

Table 2 ARV drugs default rate among HIV patients

\begin{tabular}{lll}
\hline Default rate & Percent & Frequency \\
\hline Always Consistent & 71.5 & 186 \\
Consistent sometimes & 15.8 & 41 \\
Not consistent & 9.6 & 25 \\
No response & 3.1 & 8
\end{tabular}

Source: Field Survey, 2019

Our study reveals that the most common antiviral combination therapy for the management of HIV infection among PLWHA in the Northern and Bono Regions of Ghana is the Tenofovir-LamivudineEfavirenz combination. This therapy combines two nucleoside reverse transcriptase inhibitors (Tenofovir and Lamivudine) and one non-nucleoside reverse transcriptase inhibitor (Efavirenz).

\section{ARV drug default rate by HIV patients}

The default rate of antiretroviral medication among the study subjects is presented in Table 2. Majority of the study participants $(71.5 \%)$ were always consistent with their medication. Again, about $16 \%$ indicated that they were sometimes consistent with their medication. However, about $10 \%$ of the study subjects were not consistent in taking their ARV drugs.

\section{Relationship between ARV default rate and HIV viral load}

The relationship between ARV default rate and HIV viral load was also explored. As indicated, the mean viral load among patients who were always consistent with the ARV was $49571.69 \pm 30548.89$ copies/ $\mathrm{mL}$ and those who were consistent sometimes was $72566.24 \pm 23877.81$ copies/mL while s defaulter was $845334.38 \pm 409285.62$ copies/ $\mathrm{mL}$. The mean HIV load for adherents compared to those who were sometimes consistent was not significantly different ( $p$ value $=0.969$ ). Adversely, comparing the means of those who were consistent defaulters was a significant value (p value $<0.01$ ) (Table $3 \&$ Figure 2).

Table 3 ARV drug default rate among HIV patients in the different study areas

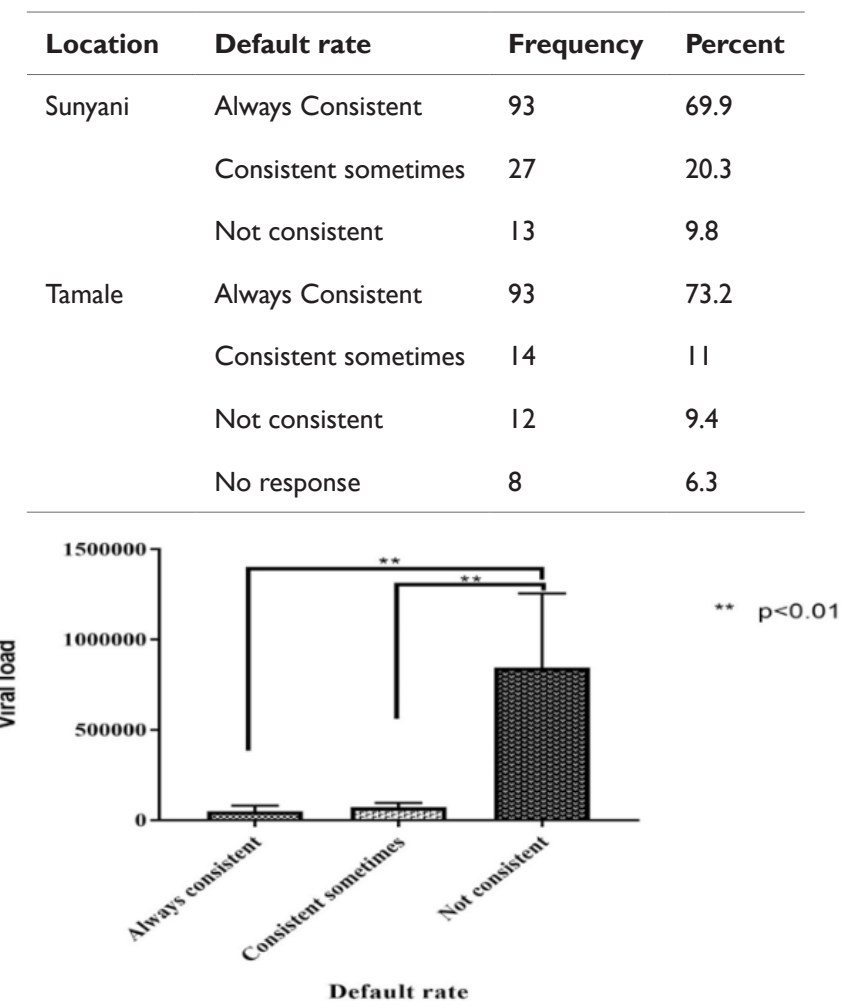

Figure 2 Comparison of antiretroviral adherence rates.

\section{Hematological parameters of participants}

The hematological parameters among participants with their attitudes towards HAART are shown in Tables $4 \& 5$. The hematological parameters that were estimated included hemoglobin levels (g/ 
dL), white blood cell (WBC), lymphocyte, monocyte, eosinophil, neutrophils, and basophil counts. No statistically significant differences were observed in all the hematological parameters analyzed between adherents and defaulters of HAART except in the hemoglobin level. The difference between hemoglobin levels of 12.04, $\mathrm{SD} \pm 0.17$, among PLWHA who adhered to HAART and 10.51, $\mathrm{SD} \pm 0.60$, among defaulters of HAART was statistically significant with a p-value of 0.007 .

Table 4 Mean HIV viral load among the three groups of anti-retro viral usage

\begin{tabular}{ll}
\hline Consistency of taking antiretroviral & Viral load \\
\hline Always consistent & $49571.69 \pm 30548.89$ \\
Consistent sometimes & $72566.24 \pm 23877.81$ \\
Not consistent & $845334.38 \pm 409285.62$ \\
\hline
\end{tabular}

Mean \pm Standard error

Table 5 Hematological parameters among the antiretroviral default group and nondefault group. $(\mathrm{n}=260)$

\begin{tabular}{|c|c|c|c|}
\hline & Default rate & Results & $P$ value \\
\hline \multirow[t]{3}{*}{ Hemoglobin } & Always Consistent & $12.04 \pm 0.17$ & 0.007 \\
\hline & Consistent sometimes & $11.79 \pm 0.28$ & \\
\hline & Not consistent & $|0.5| \pm 0.60$ & \\
\hline \multirow[t]{3}{*}{ WBC } & Always Consistent & $4.49 \pm 0.16$ & $0.4 \mathrm{II}$ \\
\hline & Consistent sometimes & $4.82 \pm 0.39$ & \\
\hline & Not consistent & $5.08 \pm 0.65$ & \\
\hline \multirow[t]{3}{*}{ Neutrophils } & Always Consistent & $2.66 \pm 0.46$ & 0.742 \\
\hline & Consistent sometimes & $2.21 \pm 0.29$ & \\
\hline & Not consistent & $1.87 \pm 0.32$ & \\
\hline \multirow[t]{3}{*}{ Lymphocytes } & Always Consistent & $2.22 \pm 0.4 I$ & 0.386 \\
\hline & Consistent sometimes & $2.47 \pm 0.6 I$ & \\
\hline & Not consistent & $3.75 \pm 0.79$ & \\
\hline \multirow[t]{3}{*}{ Monocytes } & Always Consistent & $0.35 \pm 0.03$ & 0.928 \\
\hline & Consistent sometimes & $0.37 \pm 0.06$ & \\
\hline & Not consistent & $0.36 \pm 0.06$ & \\
\hline \multirow[t]{3}{*}{ Eosinophils } & Always Consistent & $0.19 \pm 0.01$ & 0.484 \\
\hline & Consistent sometimes & $0.17 \pm 0.03$ & \\
\hline & Not consistent & $0.14 \pm 0.02$ & \\
\hline \multirow[t]{3}{*}{ Basophils } & Always Consistent & $0.04 \pm 0.02$ & 0.829 \\
\hline & Consistent sometimes & $0.02 \pm 0.01$ & \\
\hline & Not consistent & $0.02 \pm 0.004$ & \\
\hline
\end{tabular}

Mean \pm Standard error

\section{Comparative study of HIV load and hematological parameters among PLWHA}

Comparative analysis of HIV viral load and hematological parameters among PLWHA in the two study populations, Tamale and Sunyani, are presented in Table 6. A statistically significant difference was observed between the WBC and eosinophil counts, and hemoglobin $(\mathrm{Hb})$ levels of PLWHA in the Northern Region and the Bono Region. The WBC (P-value $=0.002)$ and eosinophil counts $(\mathrm{P}$-value $=0.015)$, and the hemoglobin levels $(\mathrm{P}$-value $=0.049)$ of PLWHA in Sunyani were higher than PLWHA in Tamale. All other hematological parameters showed no significant difference in estimation in the two study populations.

Table 6 HIV viral load and hematological parameters among patients in Tamale and Sunyani $(n=260)$

\begin{tabular}{llll}
\hline Parameters & Area & Results & P value \\
\hline Viral load & Sunyani & $\mid 36541.41 \pm 52461.23$ & 0.312 \\
& Tamale & $37693.83 \pm 15446.72$ & \\
Hemoglobin & Sunyani & $\mid 2.11 \pm 0.21$ & 0.049 \\
& Tamale & $\mid 1.54 \pm 0.19$ & \\
WBC & Sunyani & $5.09 \pm 0.19$ & 0.002 \\
& Tamale & $4.15 \pm 0.2310$ & \\
Neutrophiles & Sunyani & $2.70 \pm 0.32$ & 0.505 \\
& Tamale & $2.25 \pm 0.60$ & \\
Lymphocytes & Sunyani & $2.22 \pm 0.13$ & 0.512 \\
& Tamale & $2.65 \pm 0.63$ & \\
Monocytes & Sunyani & $0.37 \pm 0.03$ & 0.261 \\
& Tamale & $0.32 \pm 0.03$ & \\
Eosinophiles & Sunyani & $0.21 \pm 0.02$ & 0.015 \\
& Tamale & $0.15 \pm 0.02$ & 0.103 \\
Basophiles & Sunyani & $0.05 \pm 0.02$ & \\
& Tamale & $0.01 \pm 0.01$ & \\
\hline & & & \\
\hline & &
\end{tabular}

\section{Discussion}

The replication of HIV in infected persons is controlled by antiretroviral which is usually administered in combination to increase their effectiveness and reduce the occurrence of drug resistance. ${ }^{9}$ Unfortunately, many patients default in the intake of these antiretrovirals, and some completely refuse antiretroviral intake leading to a substantial variation in viral load and hematological parameters.

The most combination antiretroviral therapy administered in Tamale and Sunyani is made up of Tenofovir-Lamivudine-Efavirenz. $76.5 \%$ of participants were on this therapy. Lamivudine - Nevirapine Zidovudine was the second common antiviral combination identified in the study. ${ }^{25,26}$

areas. These antiretroviral combination therapies are in line with national recommendations for the treatment of HIV infections in ART naïve persons (Ministry of Health/Ghana Health Service, 2008). In addition to antiretroviral, hematinics and antibiotics were prescribed for PLWHA only in the Sunyani study area. These additional medications even though increase the drug burden of PLWHA are important in controlling hematological derangements and bacterial infections that are common in HIV infection. ${ }^{27-29}$ Antibiotics and hematinics were however stopped when the viral load, hemoglobin level, and risk of infection were controlled to normal levels. Our 
results revealed that a significant number (approx. 10\%) of people living with HIV/AIDS default in their adherence to HAART in the Northern and Bono Regions of Ghana. Even though the reasons for PLWHA default in their treatment regimen were not investigated in this study, the implications of default pose a serious challenge to the management and control of the disease, and prevention of resistance 30. A previous study conducted in the Korle Bu Teaching Hospital reported a default rate of $15 \%$ among PLWHA which is only slightly higher than the rate in this study. ${ }^{31}$ Defaulting persons did not take their HIV medication for more than three months. A study by the Ghana AIDS Commission in 2011, puts the mean defaulting rate in the Eastern Region of Ghana at $16.6 \%{ }^{32}$ In the same study, lack of money for transport to and from the ART site and to pay for charges at the site, and lack of food before ART is taken were the major factors contributing to the default of HAART..$^{32}$ Our results also revealed that PLWHA who are consistent or mostly consistent with their antiviral therapy had significantly reduced HIV loads in their blood compared to those who defaulted. The viral load associated with consistent or mostly consistent antiretroviral therapy was in the range of tens of thousand copies per mL while the viral load in PLWHA was in the hundreds of thousand copies per $\mathrm{mL}$ of blood. Our results are thus consistent with reports that combination therapy reduces HIV load. ${ }^{9}$ It has also been shown that the benefits derived from HIV therapy are mainly due to a reduction in viral load. ${ }^{29}$ It is important to note that, though adherence to HAART leads to the reduction of HIV loads in PLWHA, the recorded viral loads in our study also indicate that the antiviral therapy among PLWHA are consistent or mostly consistent with their treatment has not attained the viral suppression expected. Unsuppressed viral load $(>1,000 \mathrm{~mL})$ is associated with decreased survival, progression of HIV infection, and increased HIV transmission. ${ }^{33}$ Hematological manifestations are a common cause of morbidity and mortality in HIV patients. ${ }^{33,34}$ Even though our hematological parameters only revealed an elevation in lymphocytes numbers and a reduction in basophils numbers in PLWHA who default in HAART, there were no significant differences in blood cell counts between PLWHA who were consistent with HAART and those who defaulted. Mohri and colleagues reported increases in lymphocyte count in HIV infection and its reduction in antiretroviral therapy. ${ }^{3.5}$ Hemoglobin levels were however normal but significantly higher in patients who are consistently on HAART compared to defaulters. Low hemoglobin levels and anemia are common in unmanaged HIV infection and adversely affect the well-being of PLWHA. ${ }^{36}$ Finally, our results reveal that PLWHA in the Sunyani study population who are under HAART with hematinic and antibiotic supplements have significantly higher hemoglobin levels, white blood cell, and eosinophil levels than those from the Tamale study population who were under only HAART. The impact of hematinics in the management of HIV infection is still not confirmed but the potential of hematinics serving as an adjunct therapy for HIV management is still considerable. ${ }^{37}$

\section{Conclusion}

Our results support findings that default in antiretroviral combination therapy increases the HIV load in PLWHA with consequences to HIV infection management, prevention of drug resistance, and control of the infection. Defaulting in HAART also leads to lowered levels of hemoglobin compared with adherence to antiretroviral treatment. Hematinic supplements administered with antiretroviral therapy may help normalize hematological parameters especially hemoglobin levels in HIV-infected persons and reduce the impact of associated derangements.

\section{Ethics approval and consent to participate}

This study obtained ethical approval from the Institutional Review Board of the Navrongo Health Research Centre with an ethical clearance Reference ID as IRB-NHRCIRB342. Informed consent was duly obtained from all participants of this study.

\section{Consent for publication}

All authors have read the manuscript and have given their express consent for the article to be published.

\section{Acknowledgments}

None.

\section{Conflicts of interest}

The author declares that there is no conflict of interest.

\section{Funding}

None.

\section{References}

1. Zuckerman RA, Whittington WLH, Celum CL, et al. Higher Concentration of HIV RNA in Rectal Mucosa Secretions than in Blood and Seminal Plasma, among Men Who Have Sex with Men, Independent of Antiretroviral Therapy. J Infect Dis. 2004;190(1):156-161.

2. Control CfD AP. Division of HIV/AIDS prevention. Natl Cent HIV/ AIDS, viral hepatitis, Sex Transm Dis Tuberc Prev. Published online 2013.

3. Cuong DD, Thorson A, Sönnerborg A, et al. Survival and causes of death among HIV-infected patients starting antiretroviral therapy in northeastern Vietnam. Scand J Infect Dis. 2012;44(3):201-208.

4. Ankomah A, Ganle J, Lartey M, et al. ART access-related barriers faced by HIV-positive persons linked to care in southern Ghana: a mixed method study. 2016;16(1):738.

5. Barak T, Neo DT, Tapela N, et al. HIV-associated morbidity and mortality in a setting of high ART coverage: prospective surveillance results from a district hospital in Botswana. J Int AIDS Soc. 2019;22(12):e25428.

6. Walsh FJ, Bärnighausen T, Delva W, et al. Impact of early initiation versus national standard of care of antiretroviral therapy in Swaziland's public sector health system: study protocol for a stepped-wedge randomized rial. Trials. 2017;18(1):383.

7. Jones $\mathrm{DL}$, Sued $\mathrm{O}$, Cecchini $\mathrm{D}$, et al. Improving Adherence to Care Among "Hard to Reach" HIV-Infected Patients in Argentina. AIDS Behav. 2016;20(5):987-997.

8. Dowshen N, Kuhns LM, Johnson A, Holoyda BJ, Garofalo R. Improving adherence to antiretroviral therapy for youth living with HIV/AIDS: a pilot study using personalized, interactive, daily text message reminders. J Med Internet Res. 2012;14(2):e51.

9. Ngayo M, Okalebo F, Bulimo W, et al. Impact of first line antiretroviral therapy on clinical outcomes among HIV-1 infected adults attending one of the largest HIV care and treatment program in Nairobi Kenya. 2016;7(10).

10. Seeling S, Mavhunga F, Thomas A, et al. Barriers to access to antiretroviral treatment for HIV-positive tuberculosis patients in Windhoek, Namibia. 2014;3(4):268-275.

11. Kalichman SC, Katner H, Banas E, et al. Cumulative Effects of Stigma Experiences on Retention in HIV Care Among Men and Women in the Rural Southeastern United States. AIDS Patient Care STDS. 2020;34(11):484-490 
12. Ohene S, Forson Ejg. Care of patients on anti-retroviral therapy in Kumasi Metropolis. 2009;43(4):144.

13. Parker RA, Rabideau DJ, Sax PE, et al. Impact of Medication Adherence on Virologic Failure in A5202: A Randomized, Partially Blinded, Phase 3B Study. Clin Infect Dis an Off Publ Infect Dis Soc Am. 2017;64(11):1612-1614.

14. Denue BA, Kida IM, Hammagabdo A, et al. Prevalence of Anemia and Immunological Markers in HIV-Infected Patients on Highly Active Antiretroviral Therapy in Northeastern Nigeria. Infect Dis (Auckl). 2013;6:25-33.

15. Quaye W, Addai-Mensah A. Prevalence of anaemia and immunological markers among Ghanaian HAART-naïve HIV-patients and those on HAART. 2011;11(1).

16. Baroncelli S, Pinnetti C, Genovese O, Tamburrini E, Floridia Mjj. Hematological effects of zidovudine prophylaxis in newborn infants with and without prenatal exposure to zidovudine. 2011;83(3):551-556.

17. Keiser O, MacPhail P, Boulle A, et al. Accuracy of WHO CD4 cell count criteria for virological failure of antiretroviral therapy. 2009;14(10):1220-1225.

18. Sharma M, Mudimu E, Simeon K, et al. Cost-effectiveness of point-of-care testing with task-shifting for HIV care in South Africa: a modelling study. Lancet HIV. 2021;8(4):e216-e224.

19. Reepalu A, Balcha TT, Skogmar S, et al. High rates of viral suppression in a cohort of HIV-positive adults receiving ART in Ethiopian health centers irrespective of concomitant tuberculosis. $J$ Int AIDS Soc. 2014;17(4 Suppl 3):19612.

20. da Silva TP, Giacoia-Gripp CBW, Schmaltz CA, et al. T Cell Activation and Cytokine Profile of Tuberculosis and HIV-Positive Individuals during Antituberculous Treatment and Efavirenz-Based Regimens. PLoS One. 2013;8(6):e66095.

21. Xun J, Qi T, Zou L, et al. Mycobacterium tuberculosis co-infection is associated with increased surrogate marker of the HIV reservoir. AIDS Res Ther. 2020;17(1):63.

22. Ganatra SR, Bucşan AN, Alvarez X, et al. Antiretroviral therapy does not reduce tuberculosis reactivation in a tuberculosis-HIV coinfection model. J Clin Invest.2020;130(10):5171-5179.

23. (WHO). WHO. WHO Guidelines on Drawing Blood: Best Practices in Phlebotomy.Geneva World Heal Organ. Published online 2009.

24. CDC. Laboratory Procedure Manual.
25. WHO. WHO Prequalification of Diagnostics Programme PUBLIC REPORT Product: COBAS ${ }^{\circledR}$ AmpliPrep / COBAS $₫$ TaqMan ${ }^{\circledR}$ HIV1 Test, Version 2 . 0 Number: PQDx0147-046-00 Abstract Summary of Prequalification Status for COBAS ${ }^{\circledR}$ AmpliPrep / COBAS ${ }^{\circledR}$.; 2018.

26. Service $\mathrm{M}$ of $\mathrm{H} / \mathrm{GH}$. Guidelines for Antiretroviral Therapy in Ghana. National HIV/AIDS/ STI Control Programme. 2008.

27. Asiedu N, Kretchy I, Asampong E. Psycho-behavioral factors associated with neurocognitive performance among people living with HIV on antiretroviral therapy in Accra, Ghana. Afr Health Sci. 2020;20(2).

28. Ankrah DNA, Koster ES, Mantel-Teeuwisse AK, et al. Facilitators and barriers to antiretroviral therapy adherence among adolescents in Ghana. Patient Prefer Adherence. 2016;10:329-337.

29. Popper SJ, Sarr AD, Travers KU, et al. Lower Human Immunodeficiency Virus (HIV) Type 2 Viral Load Reflects the Difference in Pathogenicity of HIV-1 and HIV-2. J Infect Dis. 1999;180(4):1116-1121.

30. G Tornu E, Chipps J. Promoters and inhibitors of treatment adherence among HIV/AIDS patients receiving antiretroviral therapy in Ghana: Narratives from an underserved population. PLoS One. 2020;15(3):e0230159.

31. Wilhelm AK, Paci G, Lartey $M$, et al. Treatment default among patients initiating HAART at the Korle-Bu Teaching Hospital in Accra, Ghana. West Afr J Med.012;34(4):264-269.

32. Ashby C, Asante G, Aikins DA, et al. Factors Contributing to a.rtDefaulting in the Eastern Region, Ghana; 2011.

33. Waju B, Dube L, Ahmed M, Assefa SS. Unsuppressed Viral Load Level in Public Health Facilities: Nonvirological Predictors among Adult Antiretroviral Therapy Users in Southwestern. Ethiopia. HIV/AIDS - Res Palliat Care. 2021;13:513-526.

34. Opie J. Haematological complications of HIV infection. SAMJ, S Afr med j. 2012;102(6).

35. Mohri H, Perelson AS, Tung $\mathrm{K}$, et al. Increased Turnover of $\mathrm{T}$ Lymphocytes in HIV-1Infection and Its Reduction by Antiretroviral Therapy. J Exp Med.2011;194(9):1277-1288.

36. Harding BN, Whitney BM, Nance RM, et al. Anemia risk factors among people living with HIV across the United States in the current treatment era: a clinical cohort study. BMC Infect Dis. 2020;20(238).

37. Guwatudde D, Ezeamama AE, Bagenda D, et al. Multivitamin supplementation in HIV infected adults initiating antiretroviral therapy in Uganda: the protocol for a randomized double blinded placebo controlled efficacy trial. BMC Infect Dis. 2012;12(304). 